\title{
sciendo
}

Int. J. of Applied Mechanics and Engineering, 2021, vol.26, No.3, pp.187-197

DOI: 10.2478/ijame-2021-0044

\section{THREE-BLADED HORIZONTAL AXIS WATER TURBINE SIMULATIONS WITH FREE SURFACE EFFECTS}

\author{
L. RODRÍGUEZ ${ }^{*}$ and A. BENAVIDES-MORAN \\ Department of Mechanical and Mechatronics Engineering, Universidad Nacional de Colombia \\ Sede Bogota, COLOMBIA \\ E-mail: lerodriguezj@unal.edu.co
}

S. LAÍN

PAI+Research Group, Department of Energy and Mechanics, Universidad Autónoma de Occidente Cali, COLOMBIA

\begin{abstract}
The water level above a hydrokinetic turbine is likely to vary throughout the season and even along the day. In this work, the influence of the free surface on the performance of a three bladed horizontal-axis turbine is explored by means of a three-dimensional, transient, two-phase flow computational model implemented in the commercial CFD software ANSYS Fluent 19.0. The $k-\omega$ SST Transition turbulence model coupled with the Volume of Fluid (VOF) method is used to track the air-water interface. The rotor diameter is $D=0.8 \mathrm{~m}$. Two operating conditions are analyzed: deep tip immersion $(0.55 \mathrm{D})$ and shallow tip immersion $(0.19 D)$. Three tip speed ratios are evaluated for each immersion. Simulation results show a good agreement with experimental data reported in the literature, although the computed torque and thrust coefficients are slightly underestimated. Details of the free surface dynamics, the flow past the turbine and the wake near the rotor are also discussed.
\end{abstract}

Key words: Hydrokinetic turbine, VOF, sliding mesh, torque coefficient.

\section{Introduction}

Hydrokinetic energy is currently an active field of research, considering its potential to provide electricity to rural communities settled next to a river in developing countries (Laín, [1]; Ishak, [2]). Hydrokinetic turbines have been mostly applied to small scale generation while marine applications focus on large scale output (Muratoglu and Yuce, [3]). The main advantage of hydrokinetic technology is the possibility of obtaining energy without the need of large reservoirs, making no emissions and a minimal environmental impact. Horizontal-axis water turbines are self-starting machines and they are less prone to shaking and vibration, contrary to vertical-axis water turbines (Kirke and Lazauskas, [4]). For this reason, this study focuses on the performance of a horizontal-axis water turbine. To understand the flow dynamics and improve the turbine efficiency, computational and experimental approaches have been explored in recent years (Bahaj, [5]; Yan, [6]; Bahaj, [7]; Contreras, [8]). In that sense, Computational Fluid Dynamics (CFD) is an essential approach to analyze the fluid flow through the turbine rotor and to estimate its performance. Some numerical techniques such as blade-element-momentum (BEM) methods have been successfully employed (Batten et al., [9, 10]). Batten et al., [9] explored and validated a BEM implementation with the use of their experimental results in a cavitation tunnel and a towing tank. In a similar way, Danao et al., [11] reported turbine performance calculations with the open source blade element - momentum solver QBlade, showing acceptability and appropriateness of the model. However, BEM cannot be employed if the turbine interacts with the free surface or if the structure that holds the turbine rotor needs to be considered. Also, the wake that

\footnotetext{
${ }^{*}$ To whom correspondence should be addressed
} 
develops downstream cannot be resolved with BEM. On the other hand, three-dimensional, transient fluid flow computations provide an accurate representation of the wake downstream the rotor (Abuan and Howell, [12]).

The water level above hydrokinetic turbines can change throughout the season and even along the day. Considering the operation of a turbine in low flow and shallow rivers, the distance to the free surface can change continually in such a way as to affect the turbine overall performance. Previous numerical works have mostly ignored the effects of rotor depth, which could be very important specially for shallow-tip immersions. The main reason is the computational cost for solving two-phase flow equations. However, free-surface effects can greatly affect the overall performance so they have been considered on previous investigations (Bai, [13]; Yan, [6]; Benchikh [14], Nishi [15]). Benchikh et al., [14] investigated a Darrieus vertical axis hydrokinetic turbine under the presence of a free surface using the volume of fluid (VOF) method and the unsteady $k-\omega$ SST turbulence model. Their two-phase flow simulation results showed little discrepancy with experimental data of Koshizuka et al., [16]. Their results also showed that single-phase flow computations (in the absence of a free surface) overestimate the power coefficient. Bai et al., [13] used an immersed boundary method and a free surface method with extensive validation cases including the water turbine of Bahaj et al., [5]. The reported discrepancy between simulation results and measurements of the power coefficient were about $4 \%$ for deep tip immersion. Yan et al., [6] presented a computational free-surface flow framework and a novel level-set re-distancing procedure with an excellent agreement related to experimental results. In general, simulation results show a reduction in the power coefficient when the rotor is close to the free surface, which agrees well with experimental observation, except for the results reported by Nishi et al., [15]. Also, a power enhancement zone has been reported which suggests an optimal distance from the free surface (Kolekar et al., [17]). It is expected from previous works that single-phase flow simulations predict a similar performance to deep tip immersion configurations.

The hydrodynamic performance of a three-blade horizontal axis hydrokinetic turbine is studied in this work by means of numerical simulations. The computational model considers free surface effects, i.e. the airwater interface. The turbine geometry is taken from the literature [5] which also reports experimental data. Two immersions: deep tip immersion $(0.55 D)$ and shallow tip immersion $(0.19 D)$ were simulated, each one at three tip speed ratios (TSR): 4, 6 and 8 . This work deals with transient simulations incorporating a Transition Shear Stress Turbulence Model. To the best of authors knowledge, it is the first time such a turbulence model, coupled with VOF, is used in numerical simulations of hydrokinetic turbines. A quantitative description of the free surface displacement and the asymmetry of the rotor wake are the main contributions of this work to the current state of the art on the simulation of horizontal axis river turbines.

This paper is organized as follows: In section 2, the turbine layout, the computational domain and its discretization are described. Section 3 provides a detailed description of the computational model. The computed performance coefficients and the discussion of results is found in section 4 . Finally, the last section presents the conclusions and future work.

\section{Geometry and discretization}

The rotor diameter of the simulated hydrokinetic turbine is $D=0.8 \mathrm{~m}$. The blades are based on the profile shape of a NACA 63-8XX, and the information about the chord, thickness and pitch was taken from [5]. The computational domain consists of two sub-domains: a rotating part and a steady one. The rotating domain has the shape of a cylinder and includes the turbine rotor. $86 \%$ of the cells in the computational domain are found in the rotating cylinder; mesh refinement is necessary along the rotor blades in order to fulfill the near wall treatment. Fifteen layers with an inflation of 1.2 were set in ANSYS Meshing 19.0 to mesh the blades and hub walls. The static domain is prismatic with an inner hole to fit the rotating domain. Different mesh sizes were employed in order to improve the resolution of the domain interfaces, the downstream wake and the free surface interface. To ensure mesh quality, skewness was checked to be less than 0.78 all over the domain with an average value of 0.28 in all meshes used.

The size of the computational domain is parameterized in terms of the rotor diameter, $D$ : length $10 D$, height and width $3.5 D$, with the rotating sub-domain located at $3 D$ from the domain inlet, as it is shown in Fig.1. 
The blockage ratio, defined as the ratio of rotor swept area to the channel cross-sectional area (Daskiran et al. [18]), is $6.4 \%$ when the domain is filled up with water, $8.01 \%$ for deep tip immersion $(0.55 D)$ and $9.2 \%$ for shallow tip immersion (0.19D). In spite of having quite high blockage ratios, it has been reported that blockage ratios below $10 \%$, do not significantly affect the turbine performance, thus no blockage corrections are considered (Kolekar et al., [19]).

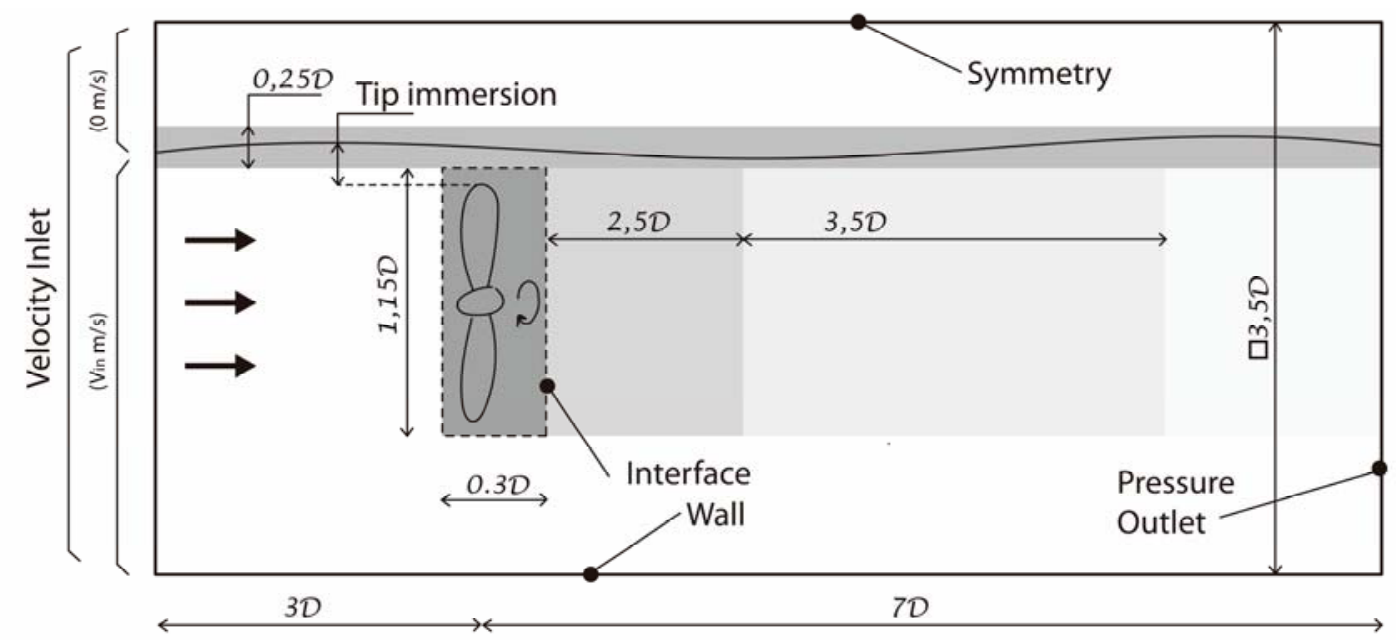

Fig.1. Schematic of the computational domain and boundary conditions. The domain height and width is 3.5D. Dark areas highlight mesh refinement zones.

\section{Computational approach}

Transient simulations were computed in ANSYS 19.0. In order to simulate the rotor rotation, one portion of the domain rotates at a constant angular velocity by means of a sliding mesh technique. In that approach, the cylindrical sub-domain that contains the rotor and hub rotates while the interface between rotating and static sub-domains is updated at every time step. The volume of fluid (VOF) method was used to track the air-water interface. VOF computes the volume fraction of one phase (i.e. water) to follow the free surface deformation (Koshizuka, [16]; Benchikh, [14]) The solved transport equations are: equation of mass, equation of momentum and equation of volume conservation, respectively (Nishi et al., [15])

$$
\begin{aligned}
& \frac{\partial}{\partial t}\left(\alpha_{W} \rho_{W}\right)+\frac{\partial}{\partial x_{j}}\left(\alpha_{W} \rho_{W} v_{j}\right)=0 \\
& \frac{\partial}{\partial t}\left(\rho v_{i}\right)+\frac{\partial}{\partial x_{j}}\left(\rho v_{i} v_{j}\right)=\frac{\partial P}{\partial x_{i}}+\frac{\partial}{\partial x_{j}}\left[\mu\left(\frac{\partial v_{i}}{\partial x_{j}}+\frac{\partial v_{j}}{\partial x_{i}}\right)-\rho \overline{v_{i}^{\prime} v_{j}^{\prime}}\right]+f_{i}+\rho g_{i} \\
& \alpha_{W}+\alpha_{A}=1
\end{aligned}
$$

where subscripts $W$ and $A$ refer to water and air phases, respectively; $i$ and $j$ are indices, $f$ is the external body force per unit volume. The Reynolds stresses $\rho \overline{v_{i}^{\prime} v_{j}^{\prime}}$ are computed according to the turbulence models described below. Furthermore, the viscosity $\mu$ and the fluid density $\rho$ are given by, 


$$
\begin{gathered}
\mu=\alpha_{W} \mu_{W}+\alpha_{A} \mu_{A}, \\
\rho=\alpha_{W} \rho_{W}+\alpha_{A} \rho_{A} .
\end{gathered}
$$

The RANS $k-\omega$ Shear Stress Transport (SST) turbulence model (Menter, [20]) was initially used for the computations. In order to better resolve the flow, the four-equation transition SST model (Langtry and Menter, [21]) was implemented. Most of the single-phase simulations and all of the two-phase flow computations were performed with the transition turbulence model. This approach is known to improve the accuracy of turbine performance computations compared to two-equation eddy viscosity turbulence models (Rezaeiha et al., [22]).

The free surface reconstruction is based on the modified HRIC (High Resolution Interface Capturing scheme). It has reported good quantitative and qualitative agreement in spite of artificial effects and diffusivity (Waclawczyk and Koronowicz, [23]). Pressure-velocity coupling is achieved by the SIMPLE approach and second order upwind schemes are specified for the numerical solution. A uniform velocity of $1.5 \mathrm{~m} / \mathrm{s}$ is set at the inlet boundary for water (Bahaj et al., [5]). Air velocity was set to zero at inlet and in the air zone above the free surface, see Fig.1. Temporal discretization was chosen such that a blade rotates less than one degree per time-step, which leads to the data presented in Tab.1. for each TSR.

Table 1. Minimum and maximum time steps $(\Delta t)$ used in the two-phase flow computations.

\begin{tabular}{|c|c|c|}
\hline TSR & $\Delta t_{\min }(\mathrm{s})$ & $\Delta t_{\max }(\mathrm{s})$ \\
\hline 4 & $5 \times 10^{-4}$ & $1 \times 10^{-3}$ \\
\hline 6 & $1 \times 10^{-4}$ & $7.5 \times 10^{-4}$ \\
\hline 8 & $1 \times 10^{-4}$ & $5 \times 10^{-4}$ \\
\hline
\end{tabular}

A maximum of 50 iterations per time-step was set in order to lower the residuals and satisfy the convergence criterion of $1 \times 10^{-6}$, except for the specific turbulence frequency, $\omega$, whose residual reached $1 \times 10^{-4}$. Single-phase flow computations with the $k-\omega S S T$ turbulence model and a multiple reference frame (MRF) were first performed to initialize the computational solution. This preliminary solution was used as an initial guess in the two-phase flow computations. The air zone was patched to zero velocity and zero water volume fraction. First order schemes were used a few time steps before switching to second order schemes. The computations were run for nine seconds which leads to $21.5,32.2$ and 43.0 blade rotations for TSR 4, 6 and 8 , respectively. The non-dimensional parameters that describe the performance of horizontal axis turbines are the tip speed ratio $(T S R)$, the power $\left(C_{p}\right)$ and thrust $\left(C_{T}\right)$ coefficients. TSR relates angular velocity to the incoming fluid velocity:

$$
T S R=\frac{\Omega D}{2 V_{\text {in }}}
$$

where $\Omega$ is the rotor angular velocity, $D$ is the diameter, and $V_{\text {in }}$ is the incoming water velocity. Simulation results presented in this work were obtained for three tip speed ratios (TSR): 4, 6 and 8 . Power coefficient $\left(C_{p}\right)$ and torque coefficient $\left(C_{m}\right)$ are defined by,

$$
C_{p}=\frac{W}{\frac{1}{2} \rho V_{i n}^{3} A}, \quad C_{m}=\frac{C_{p}}{T S R}
$$


where $\rho$ is the fluid density (water in this case), $A$ is the area swept by the rotor $\left(\pi D^{2} / 4\right)$ and $W$ is the power produced by the turbine. The thrust coefficient is related to normal force acting on the rotor, $F_{T}$, and is expressed as,

$$
C_{T}=\frac{F_{T}}{\frac{1}{2} \rho V_{i n}^{2} A} .
$$

The estimated values of $C_{m}$ and $C_{T}$ by CFD are compared with experimental data taken from the literature [5].

\section{Results}

Before performing two-phase flow computations, single phase (water) simulations were run with two different turbulence models: the two equation SST $k-\omega$ model and the four-equation transition SST model. The torque coefficient calculated with the SST $k-\omega$ and four-equation transition turbulence models at TSR=6 is presented in Fig.2. These are single-phase flow results. As part of the grid independence study, three simulations were performed with the SST transition model and four additional simulations with the twoequation SST model. Data points are connected by interpolation functions in Fig.2. As it can be observed, a higher $C_{m}$ is obtained when the four-equation SST transition turbulence model is used. The computed $C_{m}$ is slightly underestimated when it is compared to the experimental result reported by Bahaj et al., [5]. A better agreement is achieved with the four-equation turbulence model due to its ability to account for the laminar-toturbulent transition on the blade surface ([Marsh, [24]; López, [25]; Langtry, [21]). On the other hand, the transition model increases the computational cost by about 30\% [24]. Figure 2 also shows that the difference between calculations of $C_{m}$ with the turbulence models is about $5 \%$ for a 8.5 million cell mesh and larger meshes. In order to improve the calculations of $C_{m}$ and $C_{T}$, the transition turbulence model is used in all twophase flow simulations with a mesh of 7.1 million cells.
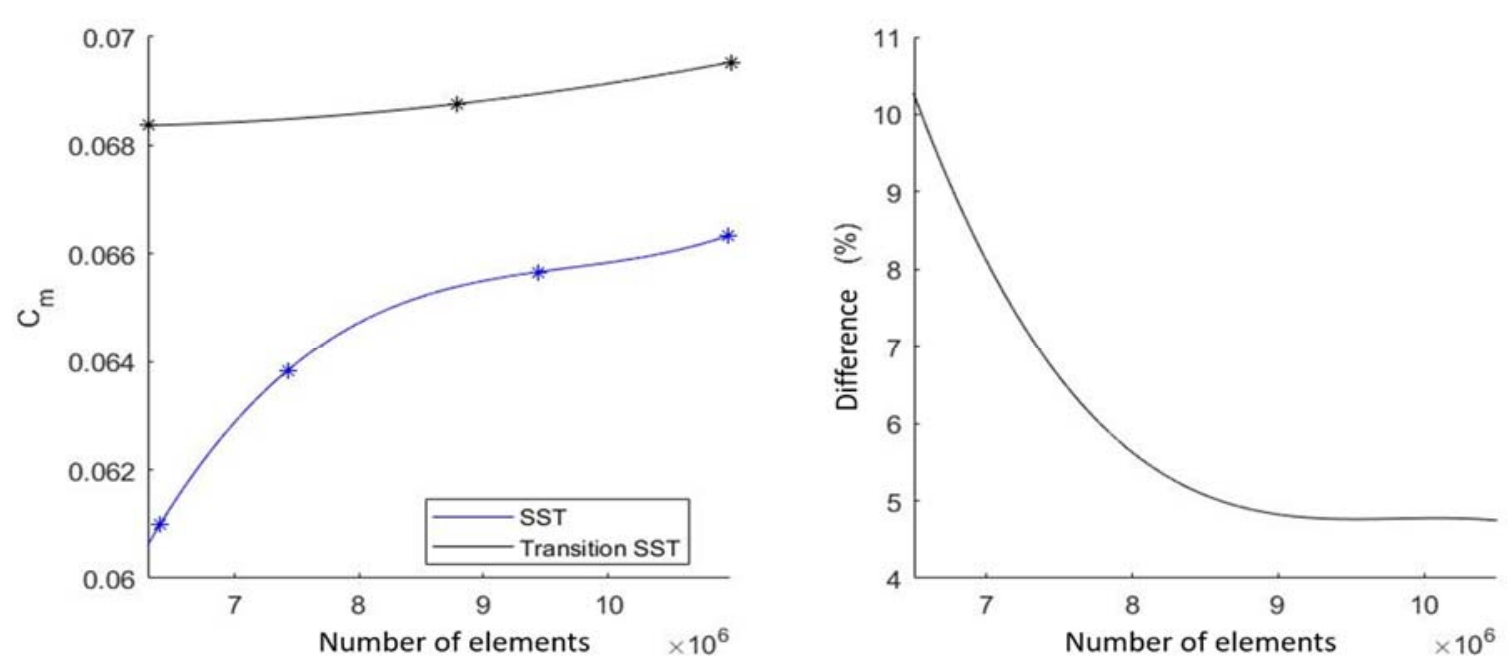

Fig.2. Sensitivity to turbulence model. Prediction of torque coefficient (left). Percentage difference between the turbulence models (right). $T S R=6$.

Figure 3 compares the computed $C_{m}$ and $C_{T}$ with the experimental data of Bahaj et al. [5] and for two rotor depths, namely deep tip immersion $(0.55 D)$ and shallow tip immersion (0.19D). In addition, MRF 
steady-state results, which correspond to single-phase computations are also included. In general, there is a good qualitative agreement between numerical and experimental data for the three analyzed TSRs; it can be observed that the computed $C_{m}$ and $C_{T}$ follow the same trend as the experiments. The torque coefficient rises a little as the rotor depth increases. The percentage difference between numerical results and experimental data goes from $13 \%$ to $22 \%$ for $C_{m}$, and $12 \%$ to $20 \%$ for $C_{T}$. The lowest discrepancy in $C_{m}$ is obtained for $T S R=6$, while for $C_{T}$ the discrepancy grows with TSR. Compared with the stationary result (MRF), a lower torque coefficient is obtained when transient calculations are performed. MRF simulations show a slightly lower thrust coefficient, although the $C_{T}$ trend looks quite similar to the transient simulation results. The numerical results show lower torque and thrust coefficients when the turbine runs close to the free surface, which agrees well with experimental observations. The highest power coefficient is obtained at $T S R=6$, i.e. the design point.
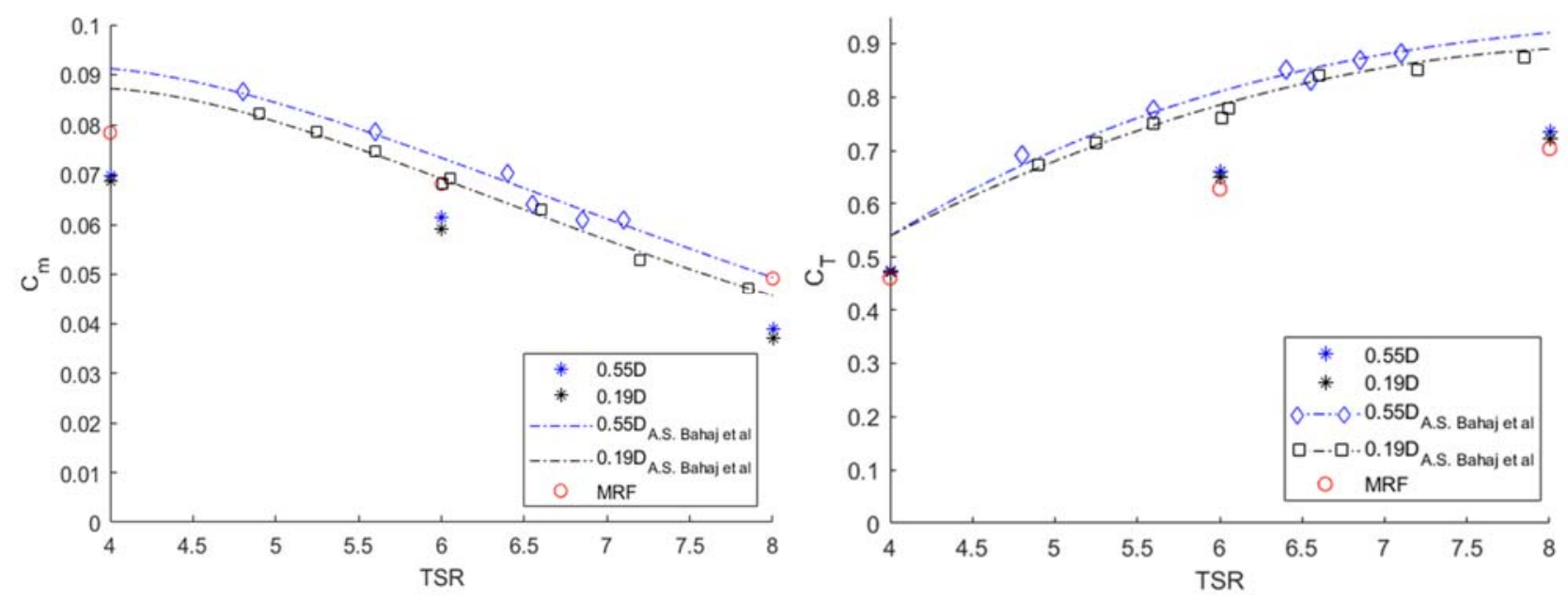

Fig.3. Time-average results of torque and thrust coefficients for three tip speed ratios and at two rotor depths. Circles represent steady-state MRF results (single-phase).
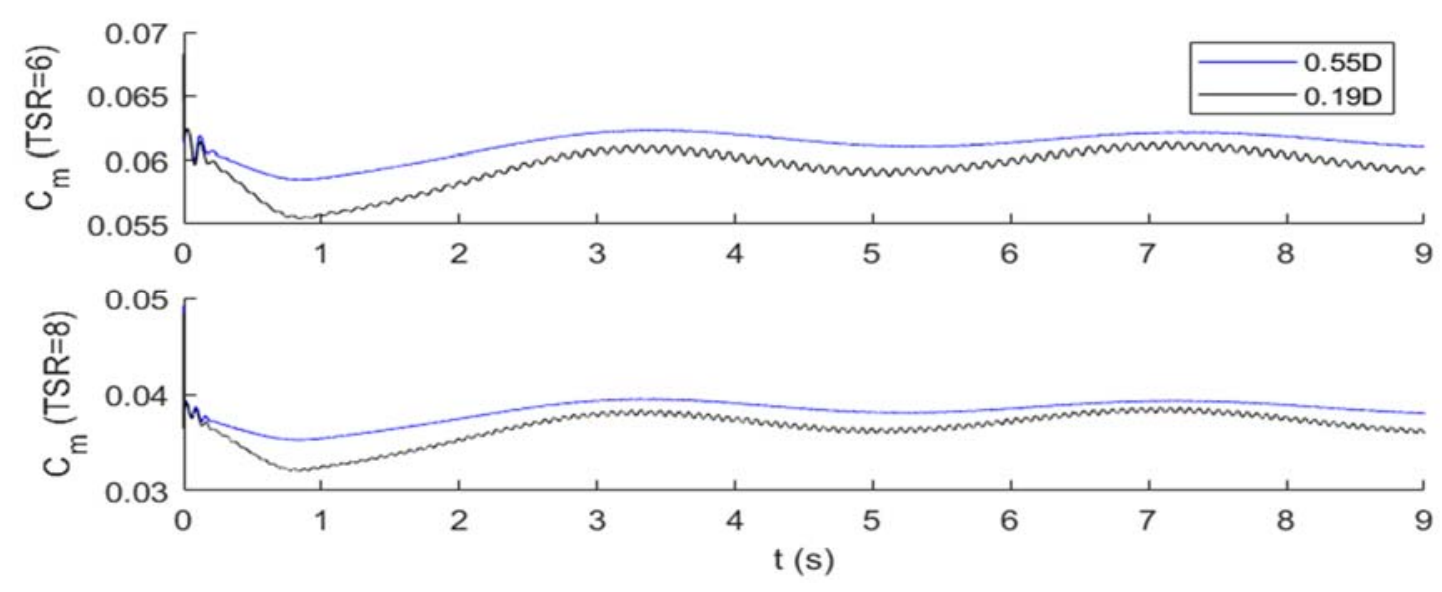

Fig.4. Transient behavior of torque coefficient at two rotor depths.

Time-average values of $C_{m}$ are obtained from transient, two-phase flow simulations. The time series is recorded over a $9 s$ period, as it can be observed in Fig.4. TSRs of 6 and 8 which correspond to 32.2 and 43.0 blade rotations, respectively. It can be noticed that high frequency oscillations are superimposed to the 
sinusoidal response of the rotor for the shallow tip immersion (0.19D). This distinctive behavior can be attributed to the proximity of the free surface. The large period of the sinusoidal wave is approximately the same for both tip immersions and TSRs. The wave amplitude is slightly higher in the case of $0.19 \mathrm{D}$ immersion. The high frequency oscillations are related to the rotor angular velocity, and therefore the TSR. That frequency is exactly three times the rotation rate of the three-bladed rotor. The low frequency oscillation of $C_{m}$ is independent of the rotor angular velocity and it is approximately the same for all the hub immersions and all the tip speed ratios simulated.

Figure 5 illustrates the variation of $C_{m}$ over one revolution, and Fig.6. shows the contribution of each blade. As it is clearly seen, the contribution of each blade to the torque coefficient combines to produce a fairly constant output for deep immersion and a roughly sinusoidal response for shallow immersion. The alternation in both, torque and thrust, decreases when increasing the immersion. When the blade tip is at the deepest position the contribution of that blade to $C_{m}$ is the highest. This feature is emphasized at shallow immersions. The alternation in torque also implies alternation in loads that could induce blade fatigue in the long run, especially for shallow tip immersions. It is important to realize that the behavior is not strictly sinusoidal as it is changing in time as observed in Fig.4. Results from single-phase flow simulations do not exhibit this variation.

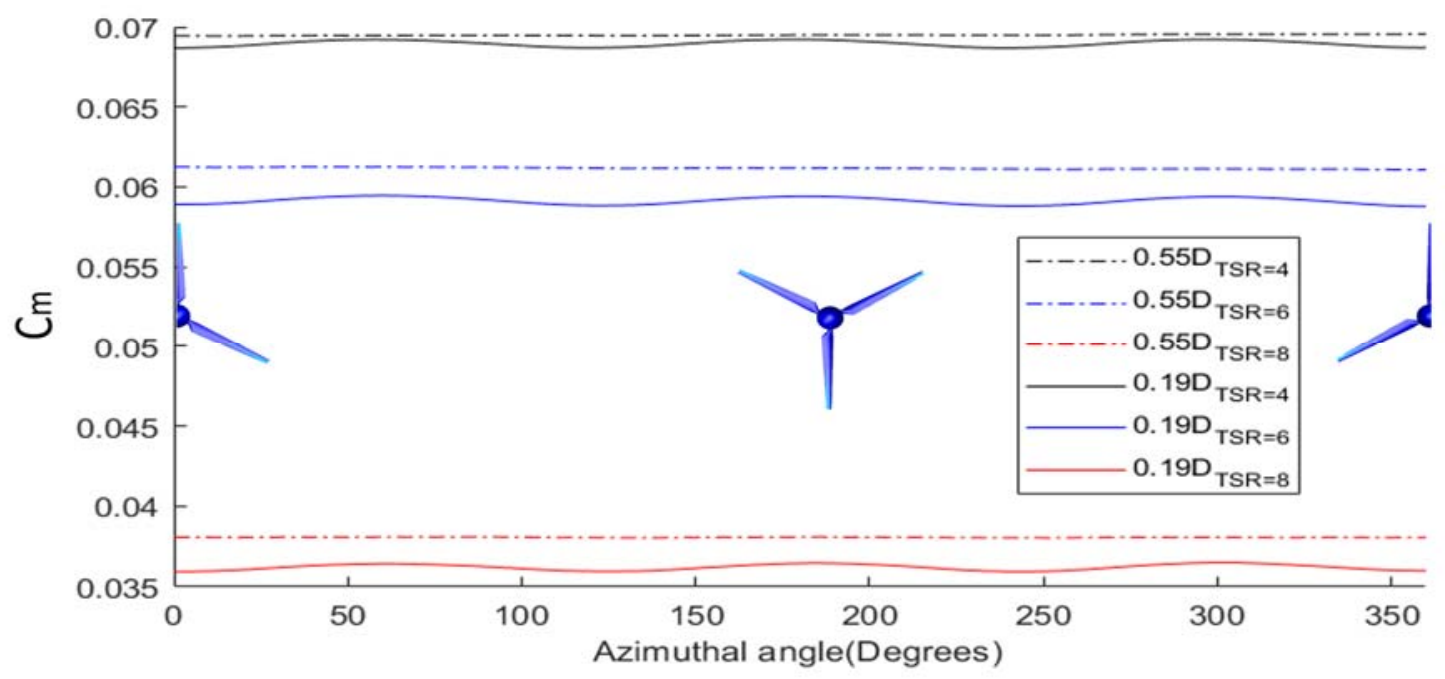

Fig.5. $C_{m}$ variation over one revolution at various TSRs and rotor depths.
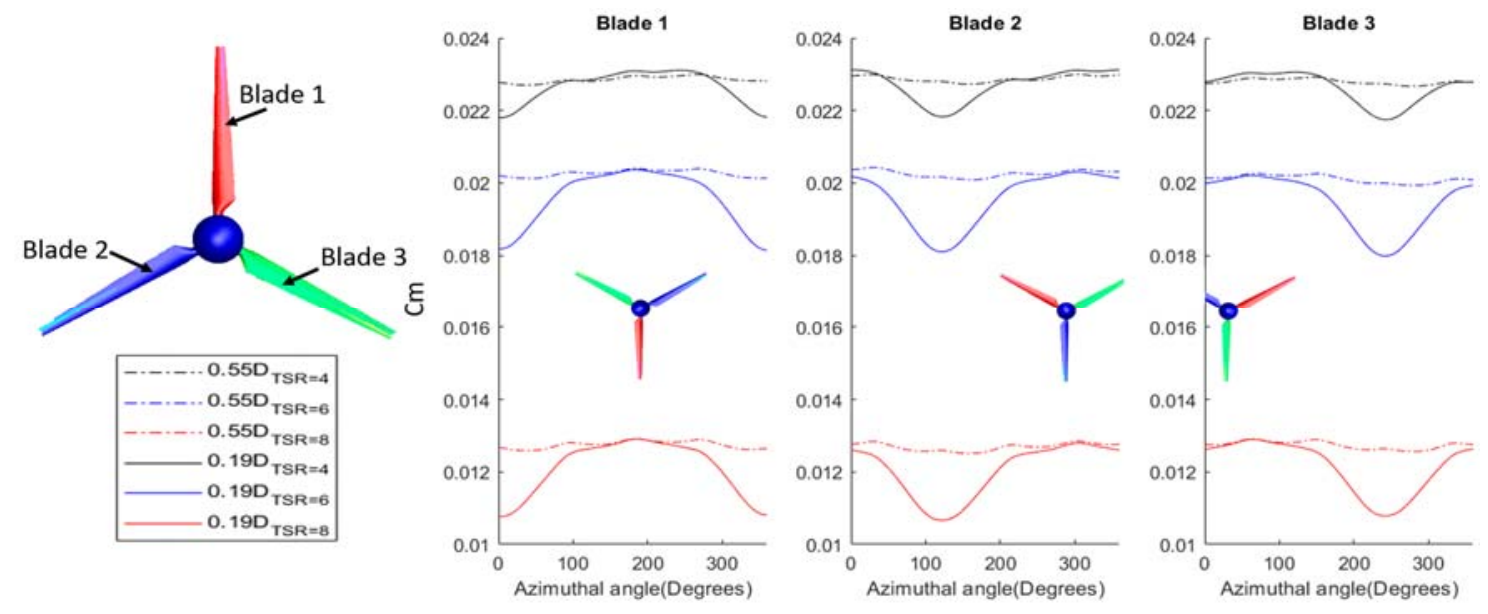

Fig.6. Blade contribution to the torque coefficient over one revolution. 
The vertical displacement of the free surface, normalized by the rotor diameter, at the domain mid-plane is shown in Fig.7. The displacement is obtained at the design tip speed ratio, TSR $=6$, and it is compared at the two rotor depths. Simulation results confirm a larger deformation just upstream and downstream the rotor for the shallow tip immersion. As the flow approaches the rotor the water slows down and its kinetic energy is modified. Some of the fluid must be diverted in order to satisfy mass conservation and the free surface must rise directly upstream of the rotor. The effect is more noticeable when the tip distance to free surface is decreased. Also, the waves formed immediately behind the rotor rapidly dissipate as the water flows downstream. The result presented in Fig.7. demonstrates the correct tracking of the free surface. It is important to mention that the height of the airwater interface is prescribed by the inlet boundary condition. Therefore, the interface may not be properly calculated in the vicinity of the inlet boundary. The free surface deformation is likely to be accentuated if the main body and upright of the turbine are included in the computational model (Myers and Bahaj, [26]).

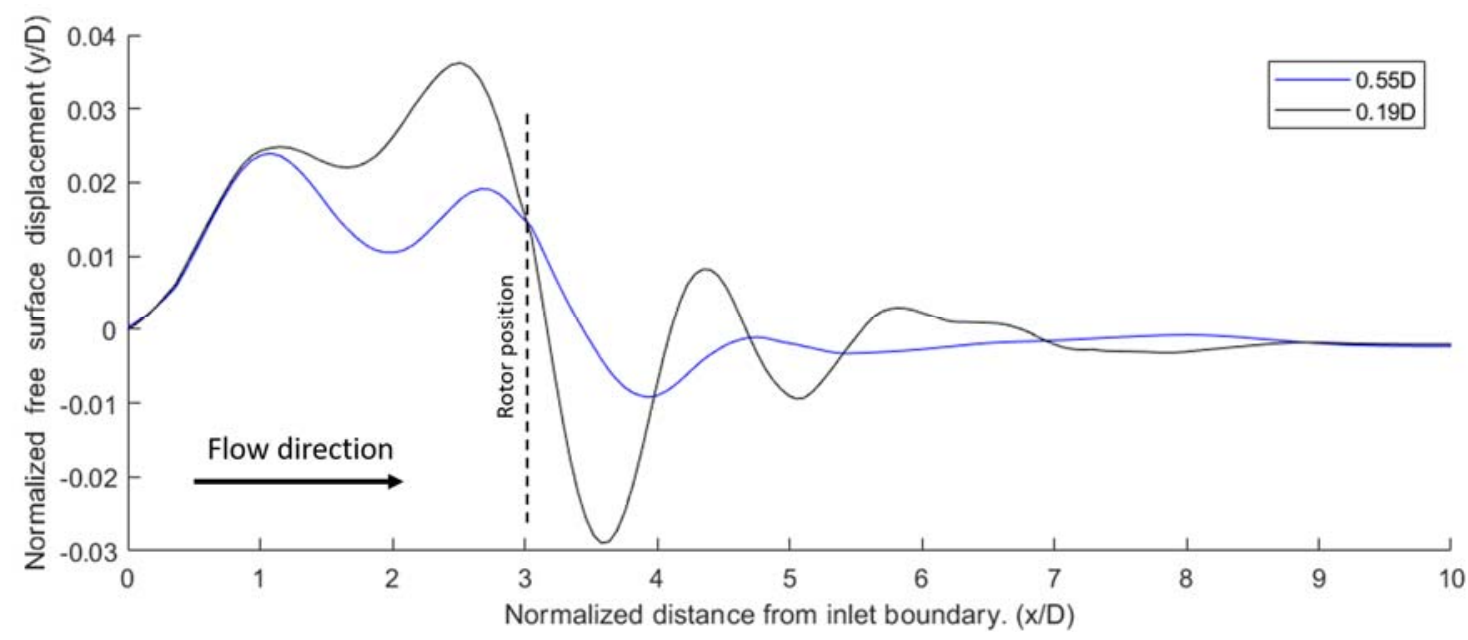

Fig.7. Vertical displacement of the free surface at the channel mid plane. Results for $T S R=6$ and two rotor depths (time $9 s)$.
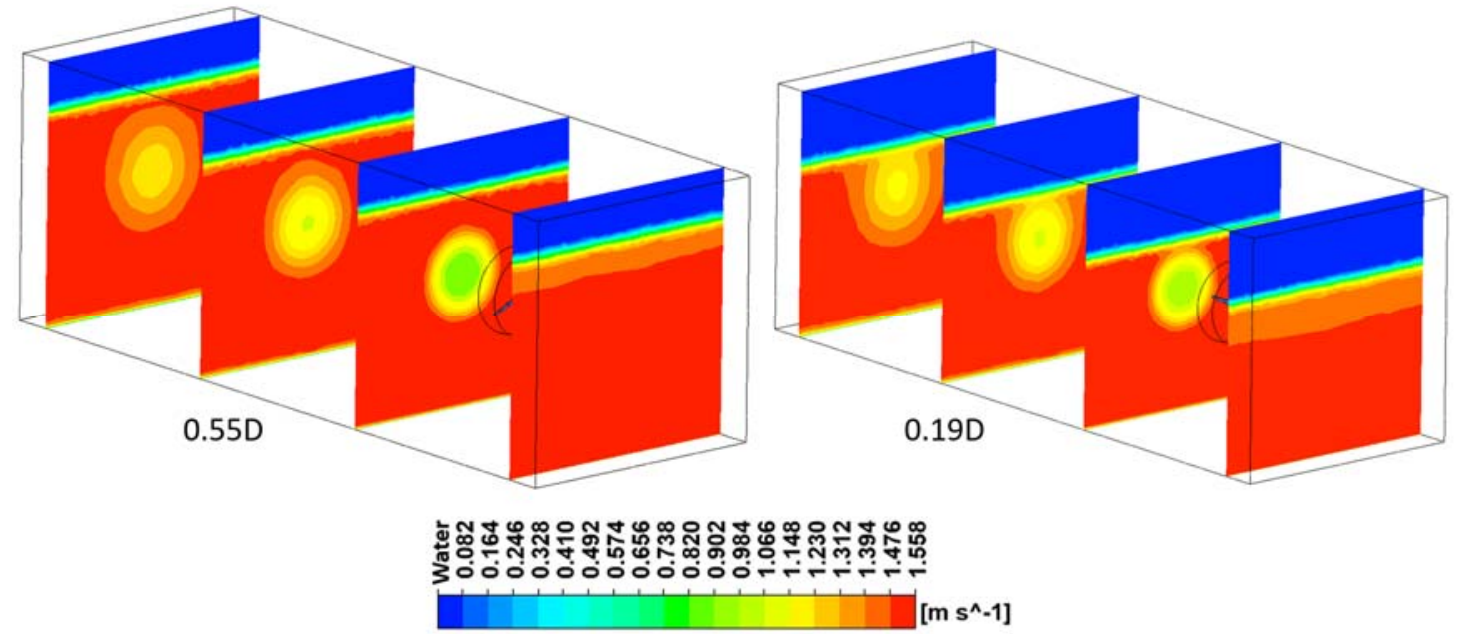

Fig.8. Axial velocity contours for rotor depths $0.55 D$ and $0.19 D(T S R=6)$.

Axial velocities in four planes ( $1 D, 4 D$ and $6.5 D$ downstream and $1 D$ from entrance) are shown in Fig.8. for the two analyzed tip immersions at $T S R=6$. The velocity deficit is clearly observed downstream. Also, non- 
symmetrical axial velocity profiles are observed when the rotor is closer to the free surface. Previous works (Adamski, [27]) have reported asymmetrical wakes for hub immersions lower than $1.5 D$, which agrees well with the results presented here. The minimum axial velocity in the wake is found off the rotor centerline and towards the free surface, contrary to single-phase flow simulations that show a symmetric wake with a minimum velocity exactly at the centerline.

\section{Conclusions}

Two-phase flow simulations of a three-bladed horizontal axis hydrokinetic turbine are presented in this paper. The computational approach includes a transition turbulence model, the VOF method for interface tracking and the sliding mesh technique to account for blade rotation. Simulation results are evaluated at three different TSR resulting in an acceptable agreement with experimental observations. The implementation of the Transition SST four-equation turbulence model leads to higher values for the torque coefficient. The time dependent behavior of $C_{m}$ shows high frequency oscillations when the rotor is close to the free surface $(0.19 D)$. It suggests that hydrokinetic turbines operating in shallow rivers are likely to be exposed to cyclic blade loads. A higher displacement of the free surface is also observed for the shallow tip immersion, and it occurs in the vicinity of the rotor. A non-symmetrical wake is observed for the shallow tip immersion which reflects the interaction between the wake and free surface.

Future work will focus on incorporating the turbine main body and upright in the computational model. The domain size and mesh quality will be further assessed. The distance from the inlet boundary to the rotor subdomain will be varied to study the effect on the free surface. Similar computations for other tip immersions will also be necessary to properly characterize the turbine performance.

\section{Nomenclature}

$$
\begin{aligned}
A & - \text { area swept by the rotor } \\
C_{m} & - \text { torque coefficient } \\
C_{p} & - \text { power coefficient } \\
C_{T} & - \text { thrust coefficient } \\
D & - \text { diameter } \\
f_{i} & - \text { external body force per unit volume } \\
F_{T} & - \text { normal force acting on the rotor } \\
\Delta t & - \text { time steps } \\
T S R & - \text { tip speed ratio } \\
v_{i} & - \text { fluid velocity in } i \text { direction } \\
V_{i n} & - \text { incoming water velocity } \\
W & - \text { power produced by the turbine } \\
\alpha_{A} & - \text { air volume fraction } \\
\alpha_{W} & - \text { water volume fraction } \\
\mu_{A} & - \text { air Dynamic viscosity } \\
\mu_{W} & - \text { water Dynamic viscosity } \\
\rho_{A} & - \text { air density } \\
\rho_{W} & - \text { water density } \\
\Omega & - \text { angular velocity }
\end{aligned}
$$




\section{References}

[1] Laín S., Contreras L.T. and Lopez O.D. (2019): A review on computational fluid dynamics modeling and simulation of horizontal axis hydrokinetic turbines.- J. Brazilian Soc. Mech. Sci. and Eng, vol.41, paper 375.

[2] Ishak Yuce M. and Abdullah Muratoglu (2015): Hydrokinetic energy conversion systems: A technology status review.Renewable and Sustainable Energy Reviews, vol.43, pp.72-82.

[3] Muratoglu A. and Yuce M.I. (2017): Design of a river hydrokinetic turbine using optimization and CFD Simulations.J. Energy Eng, vol.143.

[4] Kirke B. and Lazauskas L. (2008): Variable pitch Darrieus water turbines.- J. Fluid Sci. Technol, vol.3, pp.430-438.

[5] Bahaj A.S., Molland A.F., Chaplin J.R. and Batten W.M.J. (2007): Power and thrust measurements of marine current turbines under various hydrodynamic flow condition in a cavitation tunnel and a towing tank.- Renewable Energy, vol.32, pp.407-423

[6] Yan J., Deng X., Korobenko A. and Bazilevs Y. (2017): Free-surface flow modeling and simulation of horizontalaxis tidal-stream turbines.- Computers and Fluids, vol.158, pp.157-166.

[7] Bahaj A.S., Batten W.M.J. and McCann G. (2007): Experimental verifications of numerical predictions for the hydrodynamic performance of horizontal axis marine current turbines.- Renewable Energy, vol.32, pp.2479-2490.

[8] Contreras L.T., Lopez O.D. and Laín S. (2018): Computational fluid dynamics modelling and simulation of an inclined horizontal axis hydrokinetic turbine.- Energies, vol.11, paper 3151.

[9] Batten W., Bahaj A.S., Molland A.F. and Chaplin J.R. (2007): Experimentally validated numerical method for the hydrodynamic design of horizontal axis tidal turbines.- Ocean Engineering, vol.34, pp.1013-1020.

[10] Batten W., Bahaj A.S., Molland A.F. and Chaplin J.R. (2008): The prediction of hydrodynamic performance of marine current turbine.- Renewable Energy, vol.33, pp.1085-1096

[11] Danao L.A., Abuan B. and Howell R. (2016): Design Analysis of a Horizontal Axis Tidal Turbine.- Asian Wave and Tidal Conference.

[12] Abuan B. and Howell R. (2019): The performance and hydrodynamis in unsteady flow of a horizontal axis tidal turbine.- Renewable Energy, vol.133, pp.1338-1351.

[13] Bai X., Avital E.J., Munjiza A. and Williams J.J.R. (2014) Numerical simulation of a marine current turbine in free surface flow.- Renewable Energy, vol.63, pp.715-723.

[14] Benchikh Le Hocine A.E., Jay R.W. and Poncet S. (2019): Multiphase modeling of the free surface flow through a Darrieus horizontal axis shallow-water turbine.- Renewable Energy, vol.143, pp.1890-1901.

[15] Nishi Y., Sato G., Shiohara D., Inagaki T. and Kikuchi N. (2017): Performance characteristics of axial flow hydraulic turbine with a collection device in free surface flow field.- Renew. Energy, vol.112, pp.53-62.

[16] Koshizuka S., Tamako H. and Oka Y. (1995): A particle method for incompressible viscous flow with fluid fragmentation.- J. Comput. Fluid Dyn., vol.4, No.1, pp.29-46.

[17] Kolekar N., Vinod A. and Banerjee A. (2019): On Blockage Effects for a Tidal Turbine in Free Surface Proximity.Energies, vol.12, paper 3325.

[18] Daskiran C., Riglin J. and Oztekin A. (2016): Numerical Analysis of Blockage Ratio Effect on a Portable Hydrokinetic Turbine.- ASME International Mechanical Engineering Congress and Exposition, Phoenix, Arizona, USA

[19] Kolekar N. and Banerjee A. (2015): Performance characterization and placement of a marine hydrokinetic turbine in a tidal channel under boundary proximity and blockage effects.- Applied Energy, vol.148, pp.121-133.

[20] Menter F.R. (1993): Zonal two equation k-turbulence models for aerodynamic flows.- 23rd Fluid Dynamics, Plasmadynamics and Lasers Conference, Orlando, FL, USA.

[21] Langtry R.B. and Menter F.R. (2005): Transition Modeling for General CFD Applications in Aeronautics.- 43rd AIAA Aerospace Sciences Meeting and Exhibit, Reno, Nevada. 
[22] Rezaeiha A., Montazeri H. and Blocken B. (2019): On the accuracy of turbulence models for CFD simulations of vertical axis wind turbines.- Energy, vol.180, pp.838-857.

[23] Waclawczyk T. and Koronowicz T. (2008): Comparison of CICSAM and HRIC high-resolution schemes for interface capturing.- Journal of Theoretical and Applied Mechanics, vol.46, No.2, pp.325-345.

[24] Marsh P., Ranmuthugala D., Penesis I. and Thomas G. (2017): The influence of turbulence model and two and threedimensional domain selection on the simulated performance characteristics of vertical axis tidal turbines.Renewable Energy, vol.105, pp.106-116.

[25] López O.D, Meneses D., Quintero B. and Laín S. (2016): Computational study of transient flow around Darrieus type cross flow water turbines.- J. Renewable and Sustainable Energy, vol.8, 014501.

[26] Myers L. and Bahaj A.S. (2009): Near wake properties of horizontal axis marine current turbines.- 8th European Wave and Tidal Energy Conference.

[27] Adamski S. (2013): Numerical Modeling of the Effects of a Free Surface on the Operating Characteristics of Marine Hydrokinetic Turbines.- Thesis for master degree, University of Washington.

Received: March 31, 2021

Revised: June 19, 2021 